Reiner Keller \& Saša Bosančić

\title{
Conchita Wurst oder: Warum ich (manchmal) ein(e) Andere(r) ist
}

Macht, Subjekt, Handlungsfähigkeit - Über Erleben, Erfahren und (Auto-)Biographisieren aus Sicht der Wissenssoziologischen Diskursanalyse

„Peering from the mirror,

No, that isn't me,

Stranger getting nearer,

Who can this person be?"

(Conchita Wurst, Rise like a Phoenix, 2014) ${ }^{1}$

\section{Einführung}

Conchita Wurst alias Thomas Neuwirth gewann im Jahre 2014 mit dem Lied „Rise Like a Phoenix“ den 59. Eurovision Song Contest in Kopenhagen. Massenmedial viel beachtet wurde die als Gender-Crossing angelegte Performance, in der traditionelle männliche und weibliche Körper- und Kleidungsmerkmale kombiniert und geschlechterstereotype Zuordnungen unterlaufen wurden. Lied und passende Performance werfen Fragen nach der sozialen und diskursiven Fixierung und Auflösung von Identitäten sowie nach der Deutungs- und Handlungsmächtigkeit menschlicher Akteure auf. Diesen Fragen wollen wir uns im vorliegenden Beitrag zuwenden. Dazu möchten wir zunächst auf folgendes Beispiel rekurrieren: Auf der 2014er Tagung für International Critical Qualitative Inquiry in Urbana-Champaign stellten Richard Sawyer und Angelo Benozzo zwei sehr unterschiedliche, über die Massenmedien verbreitete (männlich-)homosexuelle Coming Out-Erzählungen vor. In der ersten Erzählung dominierte leitmotivisch die Auseinandersetzung mit den Wertvorstellungen der Tradition und Familie, sowie einer starken religiösen Orientierung. Nicht nur die Akzeptanz des eigenen 'anders seins', sondern auch deren öffentliche Erklärung setzt hier das Geständnis gegenüber und die Anerkennung durch signifikante Andere voraus: den eigenen Vater als Pater Familias, den Priester als Vertreter der religiös autorisierten Wertegemeinschaft. Dann kann der Schritt nach außen gewagt werden. Die zweite Geschichte berichtet von einem völlig anderen Verlauf. Die Selbsterfahrung, ihre Anerkennung und Veröffentlichung präsentiert sich hier als Variante des 'Yes I can' bzw. 'If you can make it (t)here, you can make it anywhere', d.h. als Realisierung und Entfaltung der Potentiale eines aktiven Ich, das sich die Welt erobert. Beide Erzählungen haben als Protagonisten Männer, die als Stars der Populärkultur im öffentlichen Rampenlicht (und auch in demjenigen der Klatschpresse) stehen: Ricky Martin und Tiziano Ferro (Sawyer/Benozzo 2014).

\footnotetext{
${ }^{1}$ Zit. nach http://www.eurovision.tv/event/lyrics?event=1873\&song=31403; Zugriff vom 13.12.2014.
} 
Schon die Schlagworte, mit denen wir die letztere der beiden Varianten belegten, deuten auf ihren kulturellen Kontext hin - diese 'Story' wurde in den USA erzählt. Im ersten Fall handelt es sich um einen Bericht aus Italien. Ein öffentliches Coming out kann also sehr unterschiedlich erlebt, erfahren und (auto)biographisiert werden; nichts garantiert, dass es nicht in unterschiedlichen Situationen eben verschieden vorgestellt wird. Es ist natürlich möglich (und wohl auch wahrscheinlich), dass in den geschilderten Fällen diese Geschichten nicht von ihren Protagonisten, sondern von cleveren Marketingfachleuten formuliert wurden - wie sage ich es meinem Publikum? Doch muss es vielleicht gar nicht um die Frage ihrer Authentizität gehen, als vielmehr darum, warum sie so und nicht anders angelegt sind - inwiefern also in ihnen eine spezifische, kulturell geprägte Art und Weise der Selbstauskunft zum Ausdruck kommt, deren Anerkennungsfähigkeit unterstellt ist. Und es ist wohl auch nicht abwegig, zu vermuten, dass sie doch genauso empfunden, erlebt, erzählt werden können, zumindest zeitweise.

Die Wissenssoziologische Diskursanalyse (WDA; z.B. Keller 2011, 2012, 2015) formuliert eine um differenzierte Akteurs- und Subjektkonzepte erweiterte Alternative zu 'poststrukturalistischen' Varianten der Diskursforschung. ${ }^{2}$ Poststrukturalistischen Perspektiven scheint mitunter eine gewisse Soziologievergessenheit zu eignen, oder eine Reduzierung der Soziologie auf struktur-funktionale Modelle (etwa eines Talcott Parsons), wie sie seit den 1950er Jahren schon soziologieintern heftig kritisiert und seit den 1980er Jahren zunehmend irrelevant wurden. Aus Sicht großer Teile der klassischen wie auch gegenwärtiger Soziologien wird die Handlungsfähigkeit menschlicher Akteure unabdingbar gesellschaftlich hergestellt, durch Prozesse der Sozialisation, die Kompetenzen der Symbolnutzung vermitteln, aber auch durch Institutionenbildungen, die Orte, Regeln und Handlungsmuster (Praktiken) zur Verfügung stellen und die die Ordnung der Wirklichkeit derart konfigurieren, dass gehandelt werden kann. Das impliziert keineswegs vollständige Determination des Handelns und der Handelnden ('Subjekte'), ebenso wenig ihr transparentes Aufgehen in Gesellschaftlichkeit, aber eben auch keine komplette 'Freiheit' des Handelns (vgl. Berger 2013 [1966]). ${ }^{3}$ Deswegen geht die Hermeneutische Wissenssoziologie mit Anklängen an den Pragmatismus davon aus, dass

\begin{abstract}
„Handlungssubjekte, hineingestellt und sozialisiert in historisch und sozial entwickelte Routinen und Deutungen des jeweiligen Handlungsfeldes, diese einerseits vorfinden und sich aneignen (müssen), andererseits diese immer wieder neu ausdeuten und damit auch 'eigen-willig' erfinden (müssen)“(Hitzler/Reichertz/Schröer 1999, S. 13).
\end{abstract}

Die Idee, dass Subjektivität, Handlungsfähigkeit und Identitäten sowohl in ihren ermöglichenden wie in ihren beschränkenden Dimensionen gesellschaftlich konstituiert sind, ist deutlich älter als das Werk Michel Foucaults und seine Studien zur Konstruktion moderner Subjektivierungsweisen, auch wenn er entsprechenden Forschungen zentrale Impulse gegeben hat. Wenn wir uns in Situationen unwohl fühlen, die 'nicht zu uns passen', Menschen begegnen, mit denen wir 'nicht klarkommen', darüber reflektieren, was uns heute widerfährt oder

\footnotetext{
${ }^{2}$ Das Etikett 'poststrukturalistisch' vereinnahmt eine Vielzahl unterschiedlicher bis gegensätzlicher Theoriepositionen. Es ist zudem missverständlich, weil alle an Foucault anschließenden Positionen der Diskursforschung (also auch die WDA) poststrukturalistisch im Sinne Foucaults sind - aber bspw. nicht im Sinne Derridas.

${ }^{3}$ Vgl. zur Komplexität von Subjekt- und Handlungsmodellen in der Hermeneutischen Wissenssoziologie Poferl/Schröer (2015).
} 
interessiert, im Unterschied oder Gleichklang zu gestern und morgen, dann beschäftigen wir uns mit identitären Stabilisierungen und Transformationen. Die eigene Identität im Plural, als Hybrid und situativ unterschiedlich zu erfahren, und sich dennoch auch mit innerlich und äuBerlich erlebbaren Kontinuitäten zu beschäftigen, ist eine alltägliche Erfahrung, und zudem ein häufiges Thema der Literatur und anderer erzählender Künste.

Die Frage nach dem mit sich identischen Subjekt, nach seiner Kontinuität und gelingenden Ich-Balance in Zeit und Raum, ist in erster Linie wohl eine Frage der Philosophie, der Psychologie und Psychoanalyse, der Pädagogik und vielleicht auch einer Literaturwissenschaft, die sich mit der Rekonstruktion der Trajekte fiktiver Figuren beschäftigt. Gewiss ist sie ein Anliegen zugreifender Institutionen, etwa der verschiedenen Religionen, aber auch politischer Ideologien und administrativer Bearbeitungsroutinen. Sie ist jedoch, wie Peter Zima (2000) vor einiger Zeit bemerkte, für die Soziologie von eher geringer Bedeutung. Diese Sichtweise liegt auch der Bestimmung des Rollenbegriffs zugrunde, die Peter L. Berger in seiner vor fünfzig Jahren, also Anfang der 1960er Jahre geschriebenen Einführung in die Soziologie, vornahm:

„Wenn man also auf die Frage, was ein Mensch im Kaleidoskop seiner Rollen 'wirklich' ist, eine soziologische Antwort geben will, so bleibt einem nichts anderes übrig, als alle die Situationen der Reihe nach aufzuzählen, in denen er irgend etwas ist.“ (Berger 1971, S. 118 [1963])

Dieser 'Mensch' bewegt sich im sozialen Gerüst differenzierter Situationen und sozialer Kreise, als im Rückgriff auf kulturelle Repertoires zur Interpretation und zum Improvisieren gezwungener Rollenspieler, als Trägerin eines situierten Handlungsvermögens und Löser von Handlungsproblemen, an die sich unterschiedliche identitäre Strategien adressieren, institutionell, sozialisatorisch und interaktiv in Kontinuitäten gezwungen und zugleich erinnerungsfähig, ein animal symbolicum, das daraus Stabilität und Orientierung gewinnt, und doch nicht darin aufgeht, subkutane Identitätsbehauptungen und „Ausbruchsversuche“ (Cohen/Taylor 1980; „I want to break free“, Queen, 1984) ${ }^{4}$ inklusive.

Diskurse und popkulturelle Symbolfiguren erweitern (oder schließen) das verfügbare, zirkulierende kulturelle Repertoire der Selbstauslegungen nicht erst seit heute, Transgender, Conchita Wurst, Madonna oder David Bowie, sondern bereits in den Filmen der 1920er Jahre (Blumer 1933), oder basaler, in den Fiktionen, Reiseerzählungen und Debatten, welche die Geschichte der Menschheit seit den oralen Kulturen begleiten. Kulturkämpfe durch die Zeitläufe hinweg sind Auseinandersetzungen über die Öffnung und Schließung solcher Repertoires, über Anerkennung und Aberkennung, über die soziohistorischen Aprioris von Handlungsfähigkeit, Macht und Subjekthaftigkeit. Wenn man von den jeweiligen spezifischen Ausprägungen im Rahmen situativer Konstellationen absieht, dann lassen sich aus wissenssoziologischer Perspektive einige allgemeine Merkmale festhalten, die den möglichen Selbstverhältnissen der Individuen und auch ihrer Handlungsfähigkeit zugrunde liegen. Gleichzeitig bietet diese wissenssoziologische Fassung der aufgeworfenen Fragen die Brücke zu diskursiven Effekten, die später diskutiert werden.

\footnotetext{
${ }^{4}$ Im Video zum Song praktizieren 1984 auch die Queen-Musiker (allen voran Freddy Mercury) GenderCrossing, das sichtbar einen (hier Schnauz-)Bart mit weiblicher Kleidungs- und Körperästhetik koppelt.
} 
Zunächst beschäftigen wir uns deswegen im Folgenden mit der Unterscheidung von Erleben und Erfahrung in der Grundlegung der menschlichen Handlungsfähigkeit, sowie mit der Rolle, die gesellschaftliche Wissensvorräte dabei spielen. Im Anschluss daran diskutieren wir die damit verbundenen Fragen der „Handlungsmacht“ (agency) und „Widerständigkeit“ und plädieren für ein minimal-anthropologisches Verständnis der menschlichen Weltbeziehungen. In Abschnitt vier wird der theoretische Zusammenhang von Diskursen, Subjekten und Biographien mit den Akteurskategorien der WDA erläutert, bevor wir im letzten Schritt der Argumentation diskutieren, inwiefern das hier vorgeschlagene Konzept der Subjektivierung, das sich an den 'Rändern' der Diskursanalyse bewegt, Anschlussmöglichkeiten für die Biographieforschung eröffnet.

\section{Wissensgrundlagen von Handlungsfähigkeiten, Selbstverhältnissen und (Auto- )Biographisierungen}

In seinen sozialphänomenologischen Analysen der Sinnkonstitution im menschlichen Leib/Bewusstsein unterscheidet Alfred Schütz (1974) im Kontext der Phänomenologie zwischen Erleben und Erfahren. Erleben ist die permanente, immer leibgebundene Verarbeitung von Sinnesreizen. Erfahrung ist die reflexive Zuwendung auf dieses Erleben. Während Erleben unserem Selbst nicht bewusst zugänglich ist, sondern geschieht, bezeichnet der Begriff der Erfahrung den Moment, an dem die Schwelle zur bewussten, d.h. gedanklichen Bearbeitung beginnt. Erfahrung ist dann die Markierung eines Erlebens mit einem Konzept, einem Deutungsschema (das seinerseits Teil eines strukturierten Deutungsvorrates ist), sie ist das Ergebnis von Sinnkonstitution im Einzelleibbewusstsein. Die Frage, ob diese Markierung richtig bzw. korrekt ist, lässt sich nicht theoretisch beantworten; sie zeigt sich im praktischen Vollzug bzw. in der Bewährung im situierten Handeln. Wie Erleben in Erfahrung gerinnt, bleibt uns unzugänglich. In jedem Fall setzt der Prozess Leiblichkeit und die Zusammenarbeit von Sinnlichkeit und Gedanklichkeit voraus, um zum Beispiel in einem Seminarraum Menschen von Dingen, Gesagtes von Schriftlichem, Tafeln von Äpfeln, Stimmen vom Beamerbrummen usw. zu unterscheiden. Es konstituiert dadurch den Sinn der Wirklichkeit für uns, in diesem Moment, an diesem Ort. Hier sind zwei Anmerkungen notwendig: Wenn wir von Sinn oder Sinnhaftigkeit sprechen, meinen wir nicht, dass es sich um etwas 'Sinnvolles' oder gezielt herbei Geführtes handelt. Gewiss sind wir in der Lage, Handlungspläne zu entwerfen: Wir sagen diesem Artikel zu, überlegen, ob wir in der dabei einkalkulierten Zukunft über Zeit und Konzentration verfügen werden, und irgendwann sitzen wir vor dem Blatt, dem Bildschirm. Wir entwickeln (wie Conchita Wurst) den Plan der Teilnahme am Grand Prix d'Eurovision, lassen uns einen Bart und die Haare wachsen, trainieren unsere Stimme, wählen einen KünstlerInnennamen, der viele Deutungen zulässt. Doch das sind bereits sehr elaborierte Formen der Sinnarbeit, bei der wir uns gesellschaftlicher Motivgrammatiken und vokabularien (Mills 1940) bedienen, die die basale Ebene der Sinnkonstitution im eben erläuterten Sinne voraussetzen. Und noch ein zweiter Hinweis ist wichtig: Die Fragen, wie reibungslos die Transformation von Erleben in Erfahrung von statten geht, und an welcher Stelle wir uns Erfahrung tatsächlich reflexiv vergegenwärtigen, sind nur schwer zu beantworten. Sie hängen sicherlich mit der Etappe der Sozialisationsprozesse zusammen, an der wir uns gerade 
befinden. Auch dafür wollen wir Beispiele geben: Neugeborene erlernen sukzessive im Kontakt mit signifikanten Anderen die Differenzierungen der sie umgebenden Welt, die ihnen erst einen erfolgreichen Handlungsbezug ermöglichen. Für sie stellt das Verhältnis von Erleben und Erfahrung die größte zu bewältigende Einstiegsaufgabe in ihr Dasein dar. Ähnliches geschieht Erwachsenen, wenn sie sich in unbekannten Situationen oder Handlungsprozessen befinden, etwa dann, wenn sie eine neue technikbasierte Mobilitätsform erlernen: Autofahren. Schalten, Bremsen, Gas geben, linkes Pedal, rechtes Pedal, Lenken und Blick in den Spiegel all das will koordiniert sein, und das geschieht in der Regel in einem Prozess der bewussten Körpersteuerung und permanenten Reflexion. Zumindest zu Beginn, denn dann werden die entsprechenden Bewegungen und Wahrnehmungen routinisiert, habitualisiert, sie sinken unter die Bewusstseinsschwelle aktiver Zuwendung. Wir gehen, sitzen, stehen, essen, reden, schweigen, liegen und lieben, ohne uns dies explizit im Vollzug der Tätigkeiten zu vergegenwärtigen - ja wir können all dies gerade nur in dem Maße, wie wir es nicht gedanklich steuern müssen. In diesem Sinne vollziehen wir unsere alltäglichen Routinen einfach aufgrund erfolgreicher Ein-Verleibungen.

Wenn wir (im Anschluss an Schütz) von der Sinnkonstitution im Einzelbewusstsein sprechen, meinen wir nicht, dass dieses Bewusstsein Sinn 'erfindet' bzw. ohne Außenkontakt operiert. Es kann sich üblicherweise seinen gedanklichen Zugriff auf die Welt nur begrenzt 'schaffen'. Bewusstseine arbeiten auf der Grundlage von Sozialisationsprozessen; sie sind immer schon mitkonstituiert durch soziale Strukturierungen, die aus interaktiven Prozessen menschlicher Gemeinschaften hervorgehen (Mead 1973). Schütz geht ebenfalls von der grundsätzlichen Intersubjektivität der geteilten Lebenswelt des Alltags aus: Diese ist Grundlage der Möglichkeit zur wechselseitig verschränkten Erfahrungskonstitution. Das einzelne Bewusstsein nutzt die Symbolsysteme, die ihm gesellschaftlich zur Verfügung gestellt werden - komplex strukturierte und soziohistorisch geformte ,gesellschaftliche Wissensvorräte“ (Schütz/Luckmann 1979, 1984). Mit Schütz interessiert uns die Funktion der mündlichen, schriftlichen oder gedanklichen Zeichen als Träger von Bedeutungen. Die Zeichenfunktion entsteht aus einer komplexen Verschränkung von materialem Zeichenträger (Laut, Buchstabenfolge), dessen Positionierung in einer Zeichenordnung, dem damit verbundenen weltlichen Korrelat oder Referenten, den damit verknüpften Bedeutungen, und nicht zuletzt dem konkreten Gebrauch in einer pragmatisch bestimmten Situation des Hier und Jetzt. Was hier für ganz basale Weltorientierungen festgehalten werden kann, gilt auch für stärker elaborierte Deutungsschemata, etwa für etablierte Muster der Lebenslauferzählung, in die wir schulisch eingeübt werden, zu denen Andere uns auf Partys animieren, die wir bei der PartnerInnen- oder Berufsfindung rekapitulieren, aber auch für abstraktere psychoanalytische Theorien, die uns nicht als Wortfolge beschäftigen, sondern als Erkundungs- und Erzählraster für Selbstvergewisserungen (unter Expertenanleitung), das Zusammenhänge zwischen Familienkonstellationen der Kindheit und aktuellen Verhaltensweisen herstellt (bzw. behauptet). ${ }^{5}$ Schütz spricht davon, dass das Bewusstsein im Rückgriff auf „Typisierungen“ arbeitet. Die gedanklichen Fixierungen von Erfahrung können der Singularität oder Einzigartigkeit von Phänomenen nicht gerecht werden. Sie verorten es immer als 'Beispiel von'. Typisierungen sind die Grundlage dafür,

\footnotetext{
${ }^{5}$ Peter Alheit hat früh darauf aufmerksam gemacht, wie unterschiedliche Sedimentierungen von Wissen in die biographischen Deutungen einsickern (vgl. z.B. Alheit 1990).
} 
dass wir uns in der Welt handlungsfähig orientieren können; in Sozialisationsprozessen, im Kontakt mit Menschen, Situationen und Handlungsaufgaben erwerben und vergrößern wir unseren Zugriff auf die damit gegebenen Möglichkeiten der Sinnkonstitution - sicherlich in unterschiedlicher Weise, je nach den Stationen und Kontexten unserer lebensbezogenen Verlaufskurve. Schütz spricht von einer biographischen (und sozialstrukturellen) Prägung der subjektiven Wissensvorräte. Die gesellschaftlichen Wissensvorräte, denen eine zentrale Rolle bei der Sinnkonstitution zukommt und die bei Berger/Luckmann (1980) zum Hauptgegenstand ihrer Wissenstheorie avancierten, sind immer als soziohistorisches Apriori zu begreifen. Ihre Elemente variieren in Zeit, Raum und sozialen Kontexten. Wenn massenmediale Öffentlichkeiten (bis hin zur Übertragung eines Grand Prix d'Eurovision) allgemein zugänglich sind, multiplizieren sich die Angebote der Welt- und Selbstdeutung.

\section{Interpretation, Handlungsmacht, Widerständigkeit, Freiheit}

Die vorangehend skizzierten Grundüberlegungen liegen der Handlungstheorie des Sozialkonstruktivismus zugrunde (Luckmann 1992), die, anders als ihr Kritiker aus den Reihen der Praxistheorien vorwerfen, durchaus das sozial geformte und geregelte 'Wie' des Handelns im Blick behält, und gleichwohl darauf insistiert, dass Handeln subjektiver Sinnsetzung und Motivgebung bedarf - und dass sich die Soziologie auch dafür interessieren sollte. Der Vollzug von Praktiken lässt sich zwar beobachten, aber nicht ohne nähere Kenntnis seiner Motivierungen verstehen. Das führt der kleine, empfehlenswerte Film „Kitchen Stories“ (2003; Regie Bent Hammer) ganz nebenbei vor, wenn er seinen nicht-teilnehmend beobachtenden Forscherprotagonisten dazu treibt, mit den Untersuchten zu reden, um (endlich) zu verstehen, warum im Anschluss an ein einzelnes Telefonläuten Kaffee aufgesetzt wird.

Menschen sind darauf angewiesen, ihre Situationen zu interpretieren, zu definieren, um dann zu handeln; in der Regel tun sie dies im Rahmen komplexer Interaktionsprozesse, in denen sich Situationsdefinitionen wechselseitig bestätigen, aber auch irritieren können. Der sozialisatorische Erwerb von Wissens- und Symbolvorräten, die institutionelle und organisatorische, mitunter auch dingliche Objektivierung und Regulierung von Handlungsverkettungen ermöglichen, strukturiert und begrenzt Handlungsfähigkeiten. Die 'agency' menschlicher Akteure ist Ergebnis eines komplexen Herstellungsprozesses; sie findet ihren basalen Ausdruck in der existentiellen Entkopplung von Verhalten und Situation, bzw. in der Notwendigkeit, diese Kopplung in situ herzustellen. Ob dabei von 'Freiheitsgraden' des Handelns gesprochen, und wie die 'Freiheit des Menschen' allgemein beurteilt werden kann, ist eine häufig auftauchende Frage. Folgt man Peter Berger, dann hängt ihre Beantwortung nicht von ihrem So- und SoSein, also von ihrer Essenz ab, sondern von dem Bezugssystem, von dem aus das Urteil gesprochen wird (vgl. Keller 2012). Dann kann man dennoch, wie Foucault, bestrebt sein, durch Forschung den Menschen zu zeigen, dass sie weit freier sind, als sie das üblicherweise annehmen. Zweifellos lässt sich allerdings soziologisch festhalten, dass Situationen über Ressourcenkonstellationen und Sanktionspotentiale sehr unterschiedliche Gestaltungsmöglichkeiten für ihre Definition bereitstellen. Und ebenso zweifellos kann angenommenen werden, dass die Wahrnehmbarkeit von Alternativen (der Geschlechterperformance und -ordnung, der Dar- 
stellung des Selbst etc.) die Wahrscheinlichkeiten und Möglichkeiten für andere Wahlen erhöht.

Poststrukturalistische Positionen haben handlungstheoretisch informierten Soziologien wiederholt vorgehalten, einen allzu starken, anthropologisch aufgeladenen und unhaltbaren Begriff des Subjekts, seiner Intentionen und Merkmale zu verwenden. Wir sehen das anders: Vielleicht ist in den bisher erläuterten Grundannahmen das Minimum an anthropologischen Grundannahmen über das ,,animal symbolicum“ benannt, das wir für ein soziologisches Verständnis der Zusammenhänge von Strukturen und Handlungen, des menschlichen Zwangs zur Deutung und Vermögens der Umdeutung von Situationen benötigen. Auch Foucault bestritt die Notwendigkeit solcher Annahmen keineswegs. Deswegen möchten wir ganz im Gegenteil argumentieren: All diejenigen Positionen, die sich um poststrukturalistische Subjektverständnisse bemühen, führen unter der Hand in den Argumenten der Dezentrierung starke Subjektannahmen ein, die aus unserer soziologischen Sicht heraus ihrerseits dem Gegenüber verhaftet bleiben, von dem sie sich abgrenzen wollen - einer essentialistischen Subjektphilosophie, deren Defizite Foucault gerade durch empirische Forschung korrigieren wollte (vgl. Reckwitz 2008; Keller u.a. 2005; Keller/Schneider/Viehöver 2012). Dies gilt etwa für die Versuche, Nicht-Determiniertheit, Dezentrierung, Widerständigkeit und Freiheit, das 'tatsächliche Wesen des Subjekts' aus psychoanalytischen Theorien heraus zu entfalten, etwa im Anschluss an Jacques Lacan (oder Sigmund Freud), bspw. durch die Annahme eines konstitutiven Mangels, aus dem sein 'Begehren' rührt. Zu den Argumenten gehören - etwa bei Judith Butler mit stärkeren Bezügen auf Freud und der Betonung identitärer Kontinuitäten, etwas anders bei Ernesto Laclau und Chantal Mouffe mit Bezug auf Lacan und den Fokus auf Identifikationsprozesse in diskursiven Kämpfen - in der Regel mehrere Argumente der 'Dezentrierung des Subjekts': Erstens werden Subjekte im Prozess ihrer Konstitution mit einer Art psychischem 'Rest' ausgestattet, der sich sowohl den selbstreflexiven als auch gesellschaftlichen Zugriffen entzieht (vgl. etwa Butler 2001). Zweitens ergibt sich im Zuge der Subjektkonstitution eine Leerstelle, ein 'Mangel', wodurch ein emotiv-irrationales Begehren nach Beseitigung des Mangels durch 'Andere' konstitutiv wird. Daraus folgt drittens, dass die Möglichkeiten des Subjekts zu widerständigem Verhalten nicht in diesem selbst verortet sind, sondern in den das Subjekt umgebenden 'selbstdekonstruktiven' symbolischen Sinnsystemen, oder sie ergeben sich aus den Prozessen der permanenten Verschiebung, die Jacques Derrida betonte: Jede Struktur existiere nur dadurch, dass sie performativ wiederholt wird. Jede Wiederholung sei wiederrum niemals eine exakte Kopie, sondern immer Verschiebung. Diese Annahmen zu den Bedeutungsverschiebungen haben dann Konsequenzen bei der Analyse von empirischen Seinsweisen. Was folgt daraus für das Verständnis des oder der Handelnden? Wenn die Strukturverschiebungen fortan nicht mehr primär im intendierten Deuten und Handeln der Akteure - bspw. ihrem blutig zusammengeschossenen Kampf um Anerkennung, ihren Sitzprotesten, ihren strategischen Interventionen gegen die Zumutung, 'dermaßen regiert zu werden' - verortet werden, liegt dann die Handlungsträgerschaft eigentümlicher Weise in den Strukturverschiebungen selbst? Gerade Butler (2006) ist hier freilich unentschieden, denn gleichzeitig konzipiert sie Widerstand aus psychischen Dispositionen heraus, die ihrerseits psychoanalytisch und durch transsituative Identitäten begründet sind. Demgegenüber verorten Ernesto Laclau und Chantal Mouffe emphatisch den 'eigentlichen Moment' des Subjekts in Situationen, in denen Unentscheidbarkeit in Entscheidung überführt wird. Vorher und danach existiert 
dieser Moment nicht. Wie entstehen solche Situationen? Soweit wir sehen, wird hier unter der Hand ein altbekanntes pragmatistisches Argument in Stellung gebracht: aus problematischen Situationen heraus, die Routinen des Handelns aufbrechen. Wir denken, dass ganz im Sinne Foucaults solche weitreichenden Annahmen über die Eigentlichkeit der Subjekte, ihr Begehren und die Freiheitsgrade ihres Handelns eher zum Gegenstand als zur Grundlage diskurstheoretisch informierter Forschungen gemacht werden sollten. Ganz abgesehen von dem Problem, dass die exakte Bestimmung der Grundlagen von Widerständigkeit, sollte sie denn je gelingen, alsbald gesellschaftlich auch für ihr Gegenteil genutzt werden kann. Für soziologische Forschung impliziert sie jedenfalls eine wichtige, in den entsprechenden Diskussionen kaum reflektierte Grundentscheidung: Geht es (wie bei den an Laclau \& Mouffe bzw. Butler anschließenden Forschungen) um die Frage nach einer fixierenden Erklärung des 'Warum' einer Identifikation, oder interessiert sie sich (mit Foucault) für die Präsenz ihrer Erscheinung und die davon ausgehenden Effekte?

\section{Diskurse, Subjektpositionen und Biographien}

Entgegen manch voreiliger (aber gerne wiederholter) Einschätzung interessiert sich die WDA nicht dafür, was 'in den Köpfen der Menschen' vorgeht. Sie ist auch nicht, wie die klassische sozialkonstruktivistische Wissenssoziologie, am alltagsnahen „Verstehen“ von subjektivem Handlungssinn interessiert. Gleichwohl hält sie Fragen nach dem alltäglichen Erleben und Erfahren gesellschaftlich konstruierter Wirklichkeiten für soziologisch interessant und auch etwa im Rahmen der Biographieforschung oder der Untersuchung von Lebensführungen - für durchaus mit diskurstheoretischen Überlegungen kombinierbar. Vielleicht genügt der Hinweis auf Max Webers Analyse der protestantischen Ethik, die ja ein gesellschaftliches (religiöses), diskursiv konstituierte Motivvokabular für Selbsttechnologien darstellt - und gesellschaftlich dann wirksam wird, wenn sie von Handelnden angeeignet und zur Grundlage ihres Tuns und Lassens gemacht wird.

Allerdings insistiert die WDA darauf, dass es ohne sinnhaftes Handeln keine Diskursproduktion und auch keine 'Diskurseffekte' gibt. Und die Diskursanalyse selbst ist wiederum (unweigerlich) eine sinnhafte Tätigkeit, welche ihrerseits Kompetenzen des Deutens bzw. Interpretierens nutzt. Um der Komplexität des menschlichen Faktors in Diskursen gerecht zu werden, schlägt die WDA die Unterscheidung von sozialen AkteurInnen, SprecherInnen, DiskursagentInnen, Modellsubjekten (Subjektmodelle, Subjektpositionen) und tatsächlichen Subjektivierungsweisen vor. Das ist an anderer Stelle ausgeführt (Keller 2012; vgl. auch Bosančić 2014) und soll hier nicht ausführlich wiederholt werden. Die Kategorie 'Soziale Akteure' bezeichnet potentielle KandidatInnen für diskursive Sprecherpositionen; SprecherInnen in Diskursen sind diejenigen, welche installierte Orte, also Sprecherpositionen einnehmen. Modellsubjekte sind diskursiv konstituierte Subjektschablonen, in denen Handlungsfähigkeit, Praktiken und Materialitäten auf der diskursiven Ebene miteinander gekoppelt sind. 'Tatsächliche Subjektivierungsweisen' bezeichnen empirisch rekonstruierbare Bezugnahmen, Adaptionen, Übersetzungen und 'Umwidmungen' oder Ablehnungen diskursiv und dispositiv angetragener Subjektpositionen. Darauf wird später zurückzukommen sein. 
Die WDA begreift Diskurse (und daran gekoppelte Dispositive) als spezifische Strukturierungsformen gesellschaftlicher Wissensverhältnisse und Wissenspolitiken, also der Arrangements von gesellschaftlichen Sinnverhältnissen zwischen Stabilisierung und Transformation. Das Verhältnis von Diskursen zum biographischen Erleben, Erfahren und Erzählen gestaltet sich dabei sehr komplex. ${ }^{6}$ Zunächst ist wohl davon auszugehen, dass (vergangene, andauernde) Diskurse sowie die daraus hervorgegangenen Institutionalisierungen und Dispositive große Teile der Wirklichkeit strukturieren, in die Individuen hineingeboren werden. Diesbezüglich lässt sich vielleicht davon sprechen, dass Diskurse die Kontextbedingungen des Biographischen stark (wenn auch bei weitem nicht ausschließlich) prägen. Hierbei handelt es sich um komplizierte Konstellationen, die bspw. sehr allgemeine institutionelle Strukturierungen (wie parlamentarische Demokratie; öffentliche Schulen für alle) ebenso betreffen wie konkretere soziale Beziehungen (Geschlechterverhältnisse, Erziehungsstile und dergleichen mehr). Daneben stellen die in der Flut von Diskursen unüberschaubar angebotenen Subjektpositionen oder Modellsubjekte, die mich zur Identifikation und Nachahmung oder aber zur Vermeidung (Drogenkonsum!) anrufen und vielleicht auch institutionell drängen, eine andere Form der diskursiven Intervention in Lebenspraxis dar, an der sich Individuen abarbeiten können, manchmal müssen - die von Ihnen aber auch ignoriert, unterlaufen, eigensinnig umgestaltet werden können. Schließlich bieten spezifische Diskurse auch Erzählmuster für Erinnerung, Vergangenheitskonstruktion und Selbstvergewisserungen. Das gilt nicht nur für psychoanalytische und psychotherapeutische bzw. psychologische Entwicklungstheorien, sondern auch für Formen kollektiven Erinnerns, soziologische Konstruktionen (Habitus), Religionen, Anleitungen zur Partnerfindung und weitere Ratgeberliteratur. Sie erweitern oder verengen permanent das kulturelle Repertoire (auto)biographischer Selbstvergewisserungen, einschließlich der Möglichkeit, auszuloten, ob Ich morgen ein(e) Andere(r) sein kann - und wo, wieweit, mit welchen Kosten und Belohnungen das verbunden wäre. Neben den Modellsubjekten stellten solche Erzählschablonen, aber auch unterschiedliche gesellschaftliche Institutionen und Praxen eines der Hauptinteressen der Foucaultschen Empirie der Verfahren, Wissensbestände und Legimitationen dar, welche in der beginnenden Moderne darauf ausgerichtet waren, die Wahrheit des modernen Subjekts zu erkunden und zu erschaffen, in Bezug auf seine Vernunft, seinen Körper, seine Norm- und Normierungsfähigkeit, seine Sexualitäten. Er hatte zugleich darauf hingewiesen, dass all diese Zugriffe auf das Subjekt produktive und destruktive Subjektivierungen erschaffen, erzwingen, ermöglichen, und dennoch immer wieder an ihrem Adressaten auch scheitern (Foucault 1987, 1996, 2005; Keller 2008).

Die diskursive Produktion entsprechender Subjektpositionen oder Modellsubjekte sowie daran anschließender Dispositive der Weltintervention läuft ungebrochen weiter. Eine Vielzahl diskursanalytisch orientierter Forschungen hat dafür Belege geliefert: das unternehmerische Subjekt, das kreative Subjekt, das authentische Subjekt, das selbst- oder fremdfürsorgliche Subjekt, das sexuell befreite Subjekt, das umweltfreundliche Subjekt, usw. Überwiegend handelt es sich um Analysen gesellschaftlicher Blaupausen und/oder daran anschließende dispositive Maßnahmen: die Einführung von Sanktionssystemen (Anreize und Strafen) in der Arbeitsmarktaktivierung, die Einrichtung von Mülltrennsystemen und Umweltlabeln, die Ein-

\footnotetext{
${ }^{6}$ Vgl. aus einer in Teilen ähnlichen Position heraus auch Tuider (2007) sowie die Diskussionen zum Verhältnis von Biographie und Diskurs in Völter u.a. (2005).
} 
führung von Gesundheitsaktivierung usw. Aus wissenssoziologischer Perspektive sind dies Beiträge zur gesellschaftlichen Konstruktion der Wirklichkeit in Form von Institutionenbildungen, Herstellung von Legitimationen, Wissensvorräten usw. In ihrem Bemühen um Weltorientierung greifen Handelnde, die zugleich Deutende sind, darauf zurück, um sich ihrer Welt zu vergewissern, sich zu orientieren, etwas zu tun, über sich nachzudenken, von sich zu erzählen. Einige diskursive Zumutungen stehen uns dabei im Rahmen unserer Alltagsrelevanzen ferner, anderen mag größere Bedeutung zukommen, um manche kommen wir nicht umhin (Lebensläufe in Bewerbungen; Erkundungen in Lebenskrisen; Explikationen gegenüber 'neuen Bekanntschaften').

Allerdings gehen wir davon aus, dass Diskurse nicht die einzige Instanz sind, die entsprechende Ermöglichungen und Zumutungen bereitstellen. Sicherlich spielen institutionelle Strukturierungen eine entscheidende Rolle (die wir zumindest in Teilen als Ergebnis vergangener diskursiver Prozesse begreifen können). Hinzu kommen jedoch Ereignisse, Begegnungen, Interaktionen, Gespräche, Gefühle, menschliche und nicht-menschliche Andere, die unmittelbar an konkrete Situationen angeschlossen sind. Permanent positionieren wir uns darin; und ebenso permanent werden wir positioniert. Sofern man aber an der Unterschiedlichkeit und Vielfalt entsprechender Sinngeneratoren für Handlungsfähigkeiten und Selbstverhältnisse oder Identitäten festhält (Bohn/Willems 2001; Hahn/Kapp 1987), kann von diskursiven Adressierungen nicht auf ihre Effekte geschlossen werden - vielmehr gilt es, letztere zum Gegenstand von empirischen Untersuchungen zu machen, die gleichwohl mit dem Problem konfrontiert sind, dass wir im Alltagsleben und unserer darin statthabenden permanenten „Sinn-Bastelei“ (Ronald Hitzler) in ganz unterschiedlichem Maße mit einer enormen Vielfalt entsprechender Anrufungen umzugehen haben. Gerade das macht jedoch das Interesse entsprechender Forschungen aus.

\section{Subjektivierungsweisen}

„She walked like a woman and she talked like a man (...)
Girls will be boys, and boys will be girls.
It's a mixed up, muddled up, shook up world“

(The Kinks, Lola, 1970)

Gewiss trifft es zu, dass Conchita Wurst ein Gender Crossing betreibt, das, wie bspw. auch Freddy Mercury 1984 im bereits erwähnten Queen-Video männlich konnotierte Bärtigkeit mit weiblich konnotierten Körper- und Kleiderformen sichtbar mischt (darin liegt der Unterschied sowohl zur Androgynität wie auch zur Drag-Kultur). Und sicherlich muss sier eine überzeugende Vorstellung (performance) bieten, um den Grand Prix und die anschließenden Marketingschlachten zu gewinnen. Ob es sich um eine Erfindung cleverer Strategen handelt oder der tatsächliche Ausdruck einer persönlichen Entwicklung und Entfaltung ist, das Ethos, die Haltung der Darstellung muss zum Dargestellten passen. Das hatten vor allem Erving Goffmans Arbeiten seit den 1950er Jahren immer wieder thematisiert (z.B. 1980, S. 297). Wenn von dieser Darstellung in sozialen Situationen auf die Kontinuität der Person in ihrer Selbst- und Fremdwahrnehmung geschlossen wird, lässt sich soziologisch vielleicht nach wie vor der Be- 
griff der Identität in Anschlag bringen: Anselm Strauss betonte etwa den „unabgeschlossenen, tentativen, explorativen, hypothetischen, problematischen, abschweifenden, wandelbaren und nur teilweise einheitlichen Charakter menschlicher Handlungsverläufe" (Strauss 1974, S. 97). ${ }^{7}$ In dieselbe Richtung weisen Goffmans (1980, S. 617) Analysen des Menschen als ,veränderlicher Formel", mit der man sich auf Situationen einlässt und die durch kulturelle Kontexte gesteuert wird. Das schließt die performative Aneignung gesellschaftlicher Rollen als „Verhaftung“ (Goffman 1973a, S. 99) und damit die affektiv-kognitive Bindung an die dem Selbst von außen angetragenen normativen Rollenerwartungen keineswegs aus.

Für die wissenssoziologisch-diskursanalytische Untersuchung von Identitätsangeboten (oder Subjektmodellen) bzw. stärker für die daran anschließende, den Kern der Diskursforschung jedoch verlassende Analyse von gelebten Identitäten und Subjektivierungen wird mit Mead, Goffman und anderen davon ausgegangen, dass es keinen festen „Kern der Persönlichkeit“ (Strauss 1974, S. 97) gibt, wie dies bspw. durch Eriksons (1976) Phasenmodell popularisiert wurde, an dessen Ende - das meist mit dem Ende der Adoleszenz zusammenfällt - ein 'fester Charakter', d.h. eine 'gelungene' Identität ausgebildet wird; weitere Entwicklungen sind dann nur noch Variationen des Wesenskerns. ${ }^{8}$ In der Perspektive des Interpretativen Paradigmas ist Identität u.a. ein dynamischer und unabschließbarer Prozess der Selbst-Positionierung im Kontext von Positionierungen durch Andere (konkrete Individuen, aber auch Organisationen, Institutionen und weitere nicht-menschliche Entitäten) sowie innerhalb diskursiver und kultureller symbolischer Sinnwelten (vgl. Bosančić 2014, S. 162 ff.). Positionierung bezieht sich dabei darauf, dass die sozialen Kontexte den Menschen beständig zu Anpassungsleistungen veranlassen: So wird das Individuum von anderen aufgrund des Aussehens, des Geschlechts, der Ethnie usw. identifiziert - soziale und persönliche „Identitätsaufhänger“ (Goffman 1975, S. 74), die es wie „Zuckerwatte“ (ebd.) umwickeln und haften bleiben, sich aber mitunter schnell ins Nichts hin auflösen.

Trotz des beständigen und mehr oder weniger sanften Drucks dieser alltäglichen Identitätserwartungen bewahren sich Menschen eine gewisse „Ellbogenfreiheit zwischen sich selbst und dem, womit die anderen es identifizieren möchten“ (Goffman 1973b, S. 303). Die dabei notwendigen Abweichungen von den Identitätserwartungen, etwa im Sinne von Rollendistanz, sind jedoch keine Idiosynkrasien oder 'Ausbruchsversuche aus der Gesellschaft', vielmehr gehört die Abweichung von den gesellschaftlichen Erwartungen zur 'gewöhnlichen', d.h. alltäglichen Identitätsarbeit.

Im Rahmen einer wissenssoziologisch-diskursanalytischen Perspektive lässt sich Subjektivierung demnach als ein doppelseitiger Prozess begreifen. Zum einen werden in Diskursen Subjektpositionen (verstanden als Identitätsangebote und -modelle, als Verbindungen einer situierten Handlungsträgerschaft mit 'doings', Praktiken des Handlungsvollzugs, und 'Dingen' bzw. Materialitäten, auf die sich die Praktiken beziehen) konstituiert, die an tatsächliche lebende, handelnde und verkörperte Subjekte adressiert sind und die einen gewissen Positionie-

\footnotetext{
${ }^{7}$ Entsprechende Überlegungen lassen sich bis in das erste Soziologielehrbuch der Chicago-Tradition (Park/Burgess 1921) rückverfolgen, das unter dem Eindruck der explosiven Multiethnizität Chicagos stand.

${ }^{8}$ Während Erikson diesen Wesenskern nach innen verlegt, unternimmt Bourdieu mit seinem Habitus-Konzept eine ähnliche Festlegung durch die an den gesellschaftlichen Ort gebundene Formung des Individuums in der frühen Kindheit (und nicht zufällig spricht er in Analogie zur Psychoanalyse von Sozio-Analyse).
} 
rungsdruck erzeugen (können). Zum anderen werden die so adressierten Subjekte in ihren tatsächlichen Subjektivierungsweisen vor dem Hintergrund der jeweiligen Biographie, der sozialstrukturellen Verortung und den situativ-alltäglichen Kontexten nach Maßgabe eigener, subjektiver Relevanzen auf diese 'Anrufungen' mehr oder weniger kreativ-eigensinnig reagieren, d. h. sich positionieren. Die Rekonstruktion von Subjektpositionen ist im engeren Sinne als diskursanalytische Vorgehensweise markiert, handelt es sich doch um die empirische Untersuchung von sozial situierten Rollenmustern (z. B. Vater-, Schüler-, Berufsrolle, etc.), Kollektividentitäten (z. B. geschlechtlich, religiös, ethnisch, etc.) oder Selbst-Vorgaben als Modellsubjekt bzw. ProblemverursacherIn (z. B. 'Leistungsträger vs. Arbeitslose'), die in (populär)wissenschaftlichen Spezialdiskursen, in Film und Fernsehen, in sozialen OnlineNetzwerken, in den zahlreichen (Lifestyle-)Zeitschriften und nicht zuletzt in der boomenden Ratgeberliteratur und den sich ebenso inflationär ausbreitenden Doku-Soaps prozessiert werden.

Entscheidend ist dabei, dass die in biographischen Erzählungen erfolgten Bezugnahmen auf Subjektpositionen notwendig durch „Brüche“ (Tuider 2007: 31) gekennzeichnet sind. Dies zum einen deswegen, weil die Identifikation bzw. Aneignung solcher Subjektpositionen, mag sie nun freiwillig oder erzwungen erfolgen, stets interpretationsabhängig erfolgen muss, und diese Interpretationen immer durch die in Sozialisationsprozessen konstituierten subjektiven Wissensvorräte beeinflusst sind sowie von den jeweiligen (sozialstrukturell) unterschiedlich verteilten Ressourcen abhängen. Zum anderen auch aus dem Grund einer multiplen Situiertheit moderner Individuen, die, wie unvollkommen auch immer, über ein sozial konstituiertes, aber individuell anhaftendes Erinnerungsvermögen verfügen, das um eine Vielzahl von Situationen, identitären Positionierungen usw. weiß. Gleichwohl sind solche Bezugnahmen keine allein durch die Technologien der Forschenden erzeugten Artefakte - das anzunehmen, würde den Untersuchten jede transsituative Eigenständigkeit absprechen. Zudem reicht es nicht hin, das komplexe Verhältnis von diskursiven Figurationen und biographischem Erleben und Erfahren zu analysieren, indem Interviewtexte nach einfachen 'Markern' für Diskurse und Subjektpositionen durchforstet werden, die dann bspw. als Beleg für „Subalternität“ oder „Intersektionalität" herangezogen werden. Zwar ist theoretische Sensibilität unverzichtbar, aber sie erscheint uns wenig hilfreich, wenn sie in Subsumptionslogik gerinnt. Ob und inwiefern aktuelle Diskurse bestimmte, von den Erzählenden zu eigen gemachte Zusammenhänge herstellen und so Machtwirkungen entfalten, muss in der Analyse am konkreten Material nachvollziehbar dargestellt und plausibilisiert werden. Vielleicht ist es dabei gar nicht notwendig, immer den Diskursbegriff in Anschlag zu bringen. In Thomas Schäfers und Bettina Völter (2005) exemplarisch angeführten Interviewpassagen, die den Zusammenhang von biographischen Erzählungen und Diskursen illustrieren sollen, wird bspw. dargelegt, dass die BiographIn auf den biologistischen „Abstammungsdiskurs“ (ebd.: 173) Bezug nimmt, wenn sie darüber berichtet, dass ihr Vater nicht ihr leiblicher Vater sei, und auf den Diskurs zur „Mutterliebe“, wenn Sie erzählt, dass ihre Mutter sie ,schlimm“ behandelt habe. Aus Sicht der WDA handelt es sich hierbei um ein Aufgreifen von Deutungsmustern, die als Set von Deutungen, Normen und Handlungsanweisungen in kollektiv tradierten Wissensvorräten und Motivvokabularien verankert sind (Keller 2014). Solche Deutungsmuster sind ohne Zweifel in der Vergangenheit auch Teil diskursvier Aushandlungsprozesse in wissenschaftlichen Spezialdiskursen und öffentlichen Diskursen gewesen, dadurch also über- und manchmal auch geformt - das Wissen 
um die biologische Vaterschaft ist (wohl mehr als dasjenige über soziale Vaterschaft) jedoch bereits fest im Alltagswissen verankert. In Bezug auf das (wohl nur plural zu verstehende) Deutungsmuster „Mutterliebe“ als zu erwartende Schutz- und Fürsorgebeziehung haben wir es ebenfalls mit sedimentierten Wissensbeständen zu tun. Diskursiv aktuell präsent sind eher Diskurse über Förder-, Aktivierungs-, Zukunfts- und Rollenvereinbarkeitsverwantwortung, deren diskursive Wahrheitsproduktionen auf biographische Situationen treffen. Zudem muss auch bedacht werden, dass Diskursivierungen ihrerseits (bspw. in Anerkennungskämpfen, Emanzipationsbewegungen) aus sozialer Praxis heraus entstehen können und dann vermittelt über Diskurse anschließende Praxis instruieren.

Bezüge zwischen Diskursen und biographischen Erzählungen lassen sich auf vielfältige Weise diskutieren und plausibilisieren - grundsätzlich zielt die Untersuchung von Subjektivierungsweisen im Anschluss an die WDA jedoch zum einen darauf, komplexe Anforderungsprofile an Subjekte und Identitäten in Form von diskursiv konstituierten Subjektpositionen zu rekonstruieren, und im Anschluss daran deren ebenso differenzierte, oftmals widersprüchliche und durch Brüche gekennzeichnete Aneignung in Selbst-Erzählungen zu rekonstruieren. Zum anderen unterscheidet die WDA im Anschluss an die sozialkonstruktivistische Wissenssoziologie Ebenen und Sinnbereiche kollektiver Wissensvorräte insofern, als wir davon ausgehen, dass die Beziehungen des Menschen zur Welt und zur eigenen Biographie durch symbolische Sinnsysteme unterschiedlichster Art und Genese vermittelt sind, Diskurse aber nur einen spezifischen Teil der symbolischen Ordnung ausmachen. Für das Aufweisen von Zusammenhängen zwischen Diskursen und empirischen Seinsweisen erachten wir es daher im Sinne einer Plausibilisierungsstrategie für hilfreich, diskursanalytische Studien 'vorzuschalten' (oder darauf zurückzugreifen), bevor in (biographischen) Interviews Machtwirkungen von Diskursen rekonstruiert werden. In jedem Fall besteht damit eine breitere Basis zur Plausibilisierung entsprechender Annahmen.

\section{Schluss}

Insgesamt wird im Rahmen der Wissenssoziologischen Diskursanalyse argumentiert, dass das vorschnelle Schließen von Subjektpositionen auf tatsächliche Subjektivierungsweisen die Komplexität menschlicher Lebens- und Handlungsverläufe ignoriert sowie auch die 'Wirkmacht' solcher Anrufungen überschätzt. ${ }^{9}$ Wie empirische Studien, die aus wissenssoziologisch-diskursanalytischer Perspektive Biographien und Identitäten untersuchen, dezidiert aufzeigen, kommen 'einfache' Übernahmen diskursiver Anrufungen auf der Ebene der empirischen Subjekte kaum vor, da selbst 'affirmative' und bemühte Versuche der Einnahme hegemonialer Subjektpositionen daran scheitern, dass die diskursiven Deutungsangebote einerseits abstrakt-unspezifisch und deswegen interpretationsabhängig sind, andererseits aber auch nur in der wissenschaftlichen Rekonstruktion in der Eindeutigkeit auftauchen, die für eine vollständige Übernahme notwendig wäre. ${ }^{10}$ Und letztendlich treffen diskursiv bereitgestellte Subjektpositionen und Motivvokabularien immer auf Lebewesen, die in plurale Kontexte einge-

\footnotetext{
${ }^{9}$ Vgl. dazu Graefe (2010), Junge (2008) oder Tuider (2007).

${ }^{10}$ Vgl. dazu Bosancic (2014), Pfahl (2011) und Schürmann (2013).
} 
bunden sind, an der Schnittstelle unterschiedlicher Diskurse und institutioneller Strukturierungen von Handlungsformen positioniert werden, und in ihrem Lebensvollzug sehr unterschiedlich in kollektive Wirklichkeitskonstruktionen und Wissensvorräte einbezogen sind. Sie gehen nicht in Diskursen auf (Tuider 2007, S. 28). Für die Wissenssoziologische Diskursanalyse stehen Diskurse und Dispositive (auch, im Anschluss an Foucault, solcher der Subjektkonstruktion) im Zentrum, nicht konkretes Erleben empirischer Individuen. Doch im Zusammenhang der hier diskutierten Beziehungen zwischen Biographien und Diskursen kann das durchaus ausgedehnt werden auf die Frage nach den Effekten, welche die sozial vermittelte und individuell (mehr oder weniger bewusst) getroffene 'Wahl' bestimmter Erzählungen generiert, ganz im Sinne des alten Theorems von Thomas \& Thomas: „Wenn Menschen Situationen als real definieren, sind sie real in ihren Konsequenzen“.

\section{Literatur}

Alheit, Peter (1990): Alltag und Biographie. Studien zur gesellschaftlichen Konstitution biographischer Perspektiven. Zweite, erweiterte Auflage, Bremen: Universität Bremen (Forschungsreihe des Forschungsschwerpunkts Arbeit und Bildung, Bd.42)

Berger, Peter L. (1971): Einladung zur Soziologie. München: List [1963]

Berger, Peter L. (2013): Identity as a Problem in the Sociology of Knowledge. In: Pfadenhauer, Michaela: The New Sociology of Knowledge. The Life and Work of Peter L. Berger. New Brunswick: Transaction, S. 115-126 [1966]

Berger, Peter L./Luckmann, Thomas (1980): Die gesellschaftliche Konstruktion der Wirklichkeit. Eine Theorie der Wissenssoziologie. Frankfurt am Main: Fischer [1966]

Blumer, Herbert (1933): Movies and Conduct. New York: The Macmillan Company

Bohn, Cornelia/Willems, Herbert (Hrsg.) (2001): Sinngeneratoren. Selbst- und Fremdthematisierungen in historischer Perspektive. Konstanz: UVK

Bosančić, Saša (2014): Arbeiter ohne Eigenschaften. Über die Subjektivierungsweisen angelernter Arbeiter. Wiesbaden: Springer VS

Butler, Judith (2001): Psyche der Macht. Das Subjekt der Unterwerfung. Frankfurt am Main: Suhrkamp [1997]

Butler, Judith (2006): Hass spricht. Frankfurt am Main: Suhrkamp [1997]

Cohen, Stanley/Taylor, Laurie (1980): Ausbruchsversuche. Identität und Widerstand in der modernen Lebenswelt. Frankfurt am Main: Suhrkamp [1976]

Erikson, E. H. (1976): Identität und Lebenszyklus. Frankfurt am Main

Foucault, Michel (1987): Warum ich die Macht untersuche: Die Frage des Subjekts. In: H. L. Dreyfus \& P. Rabinow: Michel Foucault. Jenseits von Strukturalismus und Hermeneutik. Frankfurt am Main: athenäum, S. 243-250 [1982]

Foucault, Michel (1996): Der Mensch ist ein Erfahrungstier. Gespräch mit Ducio Trombadori. Frankfurt am Main: Suhrkamp [1980/1978]

Foucault, Michel (2005): Foucault. In: ders., Schriften in vier Bänden. Dits et Écrits. Hg. von D. Defert u. F. Ewald. Bd. 4: 1980-1988. Frankfurt am Main: Suhrkamp S. 776-782 [1984]

Goffman, Erving (1973a): Interaktion: Spaß am Spiel/Rollendistanz. München: Piper [1961]

Goffman, Erving (1973b): Asyle. Über die soziale Situation psychiatrischer Patienten und anderer Insassen. Frankfurt am Main: Suhrkamp [1961]

Goffman, Erving (1975): Stigma. Über Techniken der Bewältigung beschädigter Identität. Frankfurt am Main: Suhrkamp [1963]

Goffman, Erving (1980): Rahmen-Analyse. Ein Versuch über die Organisation von Alltagserfahrung. Frankfurt am Main: Suhrkamp [1974]

Graefe, Stefanie (2010): 'Selber auch total überfordert'. Arbeitsbedingte Erschöpfung als performativer Sprechakt. In: Demirović u. a. (Hrsg.): Das Subjekt - zwischen Krise und Emanzipation. Münster: Westfälisches Dampfboot, S.49-64 
Hahn, Alois/Kapp, Volker (Hrsg.) (1987): Selbstthematisierung und Selbstzeugnis: Bekenntnis und Geständnis. Frankfurt am Main: Suhrkamp

Hitzler, Ronald/Reichertz, Jo/Schröer, Norbert (1999): Das Arbeitsfeld einer Hermeneutischen Wissenssoziologie. In: dies. (Hrsg.): Hermeneutische Wissenssoziologie. Konstanz: UVK, S. 9-13

Junge, Thorsten (2008): Gouvernementalität der Wissensgesellschaft. Politik und Subjektivität unter dem Regime des Wissens. Bielefeld: transcript

Keller, Reiner (2008): Michel Foucault. Konstanz: UVK

Keller, Reiner (2011): Wissenssoziologische Diskursanalyse. 3. Aufl. Wiesbaden: Springer VS

Keller, Reiner (2012): Der menschliche Faktor. Über Akteur(inn)en, Sprecher(inn)en, Subjektpositionen, Subjektivierungsweisen in der Wissenssoziologischen Diskursanalyse, in: Keller, Reiner/Schneider, Werner/Viehöver, Willy (Hrsg.): Diskurs - Macht - Subjekt. Theorie und Empirie von Subjektivierung in der Diskursforschung. Wiesbaden: Springer VS, S. 69-107

Keller, Reiner (2014): Wissenssoziologische Diskursforschung und Deutungsmusteranalyse. In: Behnke, C. u.a. (Hg.): Wissen - Methode - Geschlecht: Erfassen des fraglos Gegebenen. Wiesbaden: Springer VS, S.143-160

Keller, Reiner (2014): Assoziationen. In: Poferl, Angelika/Schröer, Norbert (Hrsg.): Wer oder was handelt? Zum Subjektkonzept der Hermeneutischen Wissenssoziologie. Wiesbaden: Springer VS, S. 67-94.

Keller, Reiner u.a. (Hrsg.) (2005): Die diskursive Konstruktion von Wirklichkeit. Konstanz: UVK

Keller, Reiner/Schneider, Werner/Viehöver, Willy (Hrsg.) (2012): Diskurs - Macht - Subjekt. Theorie und Empirie von Subjektivierung in der Diskursforschung. Wiesbaden: VS

Luckmann, Thomas (1992): Theorie des sozialen Handelns. Berlin: de gruyter

Mead, George Herbert (1973): Geist, Identität und Gesellschaft. Frankfurt am Main [1934]

Mills, C. W. (1940): Situated Actions and Vocabularies of Motive. In: American Sociological Review 5(6), S. 904-913

Park, Robert E./Burgess, Ernest W. (Hrsg.) (1921): Introduction to the Science of Sociology. Chicago: The University of Chicago Press

Pfahl, Lisa (2011): Techniken der Behinderung. Der deutsche Lernbehindertendiskurs, die Sonderschule und ihre Auswirkungen auf Bildungsbiographien. Bielefeld: transcript

Poferl, Angelika/Schröer, Norbert (Hrsg.) (2014): Wer oder was handelt? Zum Subjektkonzept der Hermeneutischen Wissenssoziologie. Wiesbaden: Springer VS.

Reckwitz, Andreas (2008): Subjekt. Bielefeld: transkript.

Sawyer, Richard/Benozzo, Angelo (2014): Cross-Atlantic Discourses in Celebrity Coming Out Stories: The Cases of Ricky Martin and Tiziano Ferro. Vortrag im Rahmen der ICQI 2014, UrbanaChampaign, Mai 2014.

Schäfer, Thomas/Völter, Bettina (2005): Subjekt-Positionen. Michel Foucault und die Biographieforschung. In: Völter, Bettina u.a. (Hrsg.): Biographieforschung im Diskurs. Wiesbaden: VS

Schürmann, Lena (2013): Schmutz als Beruf. Prekarisierung, Klasse und Geschlecht in der Reinigungsbranche. Münster: Westfälisches Dampfboot

Schütz, Alfred (1974): Der sinnhafte Aufbau der sozialen Welt. Frankfurt am Main: Suhrkamp [1932]

Schütz, Alfred/Luckmann, Thomas (1979): Strukturen der Lebenswelt. Bd. 1. Frankfurt am Main: Suhrkamp

Schütz, Alfred/Luckmann, Thomas (1984): Strukturen der Lebenswelt. Bd. 2. Frankfurt am Main: Suhrkamp

Strauss, Anselm (1974): Spiegel und Masken. Die Suche nach Identität. Frankfurt am Main: Suhrkamp [1959]

Tuider, E. (2007). Diskursanalyse und Biographieforschung. Zum Wie und Warum von Subjektpositionierungen [81 Absätze]. In: Forum Qualitative Sozialforschung 8(2), Art. 6, Adresse: http://nbnresolving.de/urn:nbn:de:0114-fqs070268

Völter, Bettina/Dausien, Bettina/Lutz, Helma/Rosenthal, Gabriele (Hrsg.) (2005): Biographieforschung im Diskurs. Wiesbaden: VS

Zima, Peter (2000): Theorie des Subjekts. Tübingen: Francke 